\title{
La collaboration entre travailleurs sociaux en milieu scolaire, enseignants et directions dans les cycles d'orientation fribourgeois : éclairages et enjeux
}

Résumé: Suite à l'arrivée des travailleurs sociaux dans les cycles d'orientation, les autorités scolaires du canton de Fribourg ont redéfini et précisé le rôle des divers professionnels de l'école. Cet article explore la pratique des travailleurs sociaux en milieu scolaire (TSS) ainsi que leur collaboration avec les autres acteurs de l'école, ceci à travers les regards croisés des TSS et des directeurs d'établissement. Les entretiens mettent en évidence la polyvalence du métier de TSS ainsi que la division du travail, les dynamiques relationnelles et les champs de tension qui apparaissent entre TSS, enseignants et directeurs.

Mots-clés: Travailleurs sociaux en milieu scolaire, enseignants, directions, cycles d'orientation, division du travail, collaboration

\section{Collaboration between school social workers, teachers and principals in the lower-secondary education in Fribourg: insights and issues}

Summary: Following the arrival of social workers in the lower-secondary education, the school authorities of the canton of Fribourg have redefined and clarified the role of the various professionals in the school. This article analyses the discourses used by school principals and school social workers (SSWs), concerning the practice of SSWs and their collaboration with other school actors. The interviews highlight the versatility of the SSWs profession as well as the division of labour, relational dynamics and areas of tension that arise between SSWs, teachers and school principals. Keywords: School social workers, teachers, school principals, lower-secondary level, division of labour, collaboration 


\section{Introduction}

Appréhender l'école comme un lieu où les problèmes personnels et sociaux des jeunes se manifestent n'est pas récent. Des collaborations entre professionnels de l'école et du travail social existent en effet depuis longtemps, sous la forme de situations d'intervention ponctuelles et spécifiques (Bohrer 2009). Toutefois, avecl'augmentation et la meilleure détection des problèmes sociaux et d'intégration scolaire des jeunes, l'école est amenée à assumer non seulement des tâches d'instruction, mais également des missions de socialisation et d'intégration (Changkakoti / Akkari 2008). L'accroissement des besoins en matière de prise en charge sociale des élèves nécessite le recours au soutien spécifique des travailleurs sociaux en milieu scolaire (ci-après TSS). L'intégration du travail social dans les écoles place ainsi au centre la question de l'articulation entre les professionnels traditionnels de l'école - enseignants, médiateurs - et les travailleurs sociaux (Bohrer 2009).

En Suisse, en particulier dans les cantons alémaniques, la pratique du travail social en milieu scolaire s'est fortement développée dans les écoles depuis les années 1990, afin d'intervenir précocement auprès des élèves en difficulté et de donner un appui aux enseignants (Bohrer 2009). Toutefois, l'organisation de l'école obligatoire étant du ressort des cantons, une grande diversité tant dans la pratique que dans les structures et concepts mobilisés par les TSS existe entre les régions.

Cet article présente quelques résultats d'une recherche exploratoire conduite dans les cycles d'orientation (CO) du canton de Fribourg sur la collaboration entre les TSS et leurs partenaires scolaires, ceci à travers les regards croisés des TSS et des directions.

\section{Contexte de la recherche}

Dans le canton de Fribourg, canton bilingue dans lequel notre recherche s'est déroulée, des postes de TSS ont été développés depuis 2010 dans les CO germanophones. Dans les écoles francophones, où leur présence est moins répandue, les travailleurs sociaux se sont installés dans un champ jusqu'alors occupé par des médiateurs scolaires (Von Aarburg 2014). L'arrivée des travailleurs sociaux a demandé à la Direction de l'instruction publique, de la culture et du sport (DICS) de repenser et renégocier les mandats et cahiers des charges confiés aux différents professionnels de l'école. En particulier, l'inscription du TSS dans la nouvelle loi sur la scolarité obligatoire et dans son règlement d'application, entrée en vigueur le $1^{\text {er }}$ août 2015 , respectivement le $1^{\text {er }}$ août 2016, a conduit à une réorganisation administrative du personnel de soutien. La médiation et le TSS font désormais 
partie du dispositif de mesures de soutien dont les établissements scolaires peuvent bénéficier dans la prise en charge des élèves présentant des difficultés de comportement (mesures SED), ce dispositif étant géré par les services de l'enseignement obligatoire de langue française (SEnOF) et allemande (DoA).

Suite aux nouvelles bases légales, les rôles, missions et aires spécifiques de responsabilités, activités et compétences de ces acteurs du soutien internes aux écoles ont été formellement clarifiés. D’une part, «Les personnes chargées de la médiation promeuvent une culture de la communication par le conseil et l'accompagnement des élèves ou des adultes (corps enseignant, parents, autres acteurs de l'école) en difficultés relationnelles.» (Règlement de la loi sur la scolarité obligatoire (RLS) du 19 avril 2016, Article 19). Ils interviennent dans des situations conflictuelles ou des difficultés de communication entre les différents partenaires de l'école (élèves, enseignants, responsables d'établissement, etc.). Les médiateurs scolaires sont des enseignants expérimentés qui ont suivi une formation continue en médiation et qui cumulent leur mandat avec celui de l'enseignement $^{1}$. D’autre part, «Les personnes chargées du travail social encouragent l'intégration des élèves à l'école et soutiennent ainsi le mandat de formation et d'éducation de celle-ci.» (Règlement de la loi sur la scolarité obligatoire (RLS) du 19 avril 2016, Article 19). Les TSS sont ainsi amenés à soutenir l'intégration de l'élève à l'école par la détection et le traitement précoce de situations personnelles ou sociales problématiques compromettant son bien-être et le bon déroulement de son parcours scolaire, en collaboration étroite avec enseignants, directions et familles. Les TSS possèdent un Bachelor of Arts HES-SO en Travail social, mais, contrairement aux médiateurs scolaires, n'ont pas suivi une formation spécifique à l'exercice de leur profession dans ce champ professionnel. Au niveau administratif, les TSS fribourgeois sont intégrés, grâce au budget des mesures SED, au sein des écoles, tout en étant indépendants du corps enseignant. Leurs attributions sont définies dans un cahier des charges élaboré en collaboration et approuvé par la direction en fonction des besoins spécifiques de l'établissement scolaire, du taux d'activité du TSS et suivant les lignes directrices intercantonales (AvenirSocial 2016). Du fait de leur intégration ${ }^{2}$ dans l'organisation de l'établissement scolaire, les TSS participent au développement et à la coordination d'objectifs et d'actions communs avec les écoles dans lesquelles ils évoluent (Kottelat 2015) et collaborent de manière directe avec les enseignants, la direction et les familles, tout en travaillant en interdisciplinarité avec d'autres professionnels internes (médiateurs, psychologues, 
etc.) et externes (unité mobile, classe relais, service de l'enfance et de la jeunesse, etc.) à l'école.

\section{Cadrage théorique}

Bien que la modification de la législation ait donné une légitimité et une reconnaissance officielle des mandats et fonctions des TSS au sein des écoles romandes (Abry Kalenga et al. 2015), il s'agit d'un champ d'intervention du travail social émergeant, encore peu défini et dont l'introduction soulève nombre d'enjeux tant professionnels (mission, cahier de charges), qu'éducatifs (compétences) et économiques (rétribution, fonction). D’une part, son introduction entraîne des incertitudes pour les TSS eux-mêmes telles qu'une attribution des responsabilités et activités variable en fonction des écoles et de leurs besoins (Kottelat 2015), une position hiérarchique à redéfinir et un rôle à clarifier et expliciter (Wattendorff / Landry 2012), une envie d'approfondir et renforcer leur spécificité (Buhler 2015) ainsi qu'un besoin de gagner en reconnaissance et visibilité auprès de l'ensemble des acteurs scolaires (Kottelat 2015). Ces incertitudes peuvent générer des tensions au sein des écoles liées notamment à la division du travail entre les TSS et les autres acteurs scolaires, au risque de concurrence et à la possible hiérarchisation entre ces divers profils professionnels (Von Aarburg 2014). En effet, les médiateurs scolaires et les enseignants peuvent vivre une potentielle remise en question de leur territoire habituel d'intervention ainsi qu'une possible redéfinition de leur activité professionnelle (Vezinat 2016). D'autre part, même si ces divers profils professionnels sont décrits et clairement différenciés d'un point de vue théorique, leur articulation dans la pratique soulève des discussions dans les milieux professionnels (i.e. établissements scolaires, AvenirSocial) relatives à la professionnalisation des TSS, aux spécificités, frontières et zones de tuilage entre enseignants, médiateurs et TSS, ainsi qu'aux rapports entre ces professionnels. Toutefois, les études empiriques qui l'abordent sont rares. En effet, des travaux de Bachelor en travail social se sont focalisés, d'une part, sur des aspects spécifiques de la pratique des TSS, tels que l'absentéisme scolaire (Cattin / Sottas 2014) ou la promotion de la santé (Chevalier 2015), d'autre part, sur la collaboration des TSS avec les familles d'élèves en difficultés (Chaignat / Jacopin 2015) ou avec des enseignants (du point de vue de ces derniers) (Aebischer 2017). A notre connaissance, une seule étude s'est intéressée au dispositif du TSS, notamment dans les établissements scolaires de la ville de Fribourg (Wattendorff / Landry 2012). Cette évaluation a mis en évidence une bonne intégration des travailleurs sociaux dans les écoles, une bonne collabora- 
tion avec les partenaires scolaires et du réseau, de même qu'une grande satisfaction par rapport à l'offre (mission adaptée aux besoins et attentes de l'école, soulagement des partenaires, etc.). Elle a également souligné la nécessité pour les TSS de clarifier leur rôle et de redéfinir leur position hiérarchique dans l'école ainsi que le besoin d'être davantage présents dans les écoles. Du côté des enseignants, la collaboration avec les TSS est peu ou pas abordée dans des travaux de recherche ${ }^{3}$. Les Hautes écoles pédagogiques sensibilisent leurs étudiants à cette question via des modules de formation consacrés aux différents intervenants et services auxiliaires (psychologues, enseignants d'appui, etc.) et prioritairement aux démarches pratiques et administratives qui permettent d'en bénéficier.

Dès lors, il nous a paru pertinent d'investiguer, au travers d'une recherche exploratoire menée dans les CO fribourgeois, la pratique professionnelle des TSS ainsi que la collaboration avec ses partenaires internes et externes à l'école et les enjeux qu'elle soulève. La présence des TSS implique-t-elle des réaménagements administratifs et organisationnels au sein des établissements scolaires? Quelles sont les missions et fonctions des TSS? Comment, par qui et sur quelle base s'opère l'attribution formelle des responsabilités et activités entre les TSS, les enseignants et les autres acteurs scolaires? Quelles sont les responsabilités et activités des TSS au quotidien? Quelles relations se développent entre les TSS et les différents acteurs de l'école et comment se passe le travail en réseau avec les familles et les services spécifiques?

Pour étudier ces questions, nous avons pris appui sur la perspective de la sociologie des groupes professionnels qui analyse principalement la dynamique desdits groupes, c'est-à-dire les processus par le biais desquels les activités professionnelles émergent, se transforment, se différencient, voire disparaissent (Bercot et al. 2012; Demazière / Gadéa 2009; Dubar et al. 2011). Ce courant se révèle pertinent dans le cadre de cette recherche pour comprendre la manière dont se passe l'introduction des travailleurs sociaux dans un nouveau champ de travail qu'est école, les responsabilités et activités qui leur sont prescrites et leur travail sur le terrain, de même que la collaboration avec des partenaires internes et externes à l'école. Ainsi, reprenant quelques questions centrales abordées par ce courant, cette recherche a investigué, dans une perspective plutôt organisationnelle, les mécanismes de recomposition de la division du travail entre TSS, médiateurs scolaires et enseignants, les mouvements dans les attributions (des fonctions, responsabilités, activités, compétences, rôles) et les spécialisations professionnelles au niveau de la prise en charge des élèves 
en difficulté, qui s'opèrent dans les CO fribourgeois à travers la collaboration entre les TSS et les autres acteurs de l'école. Cette approche nous a également permis d'explorer les rapports de concurrence et de coopération entre les TSS, les médiateurs scolaires et les enseignants, de même que les régulations formelles et informelles mises en œuvre par ces acteurs pour contrôler, défendre, voire étendre leur espace d'intervention. La question de la professionnalisation des TSS qui est habituellement étudiée par la sociologie des groupes professionnels n'a été abordée que de manière indirecte. En effet, bien que nous considérions les travailleurs sociaux comme un groupe professionnel, à savoir «des ensembles de travailleurs exerçant une activité ayant le même nom, et par conséquent dotés d'une visibilité sociale, bénéficiant d'une identification et d'une reconnaissance, occupant une place différenciée dans la division sociale du travail, et caractérisés par une légitimité symbolique.» (Demazière / Gadéa 2009, p. 20), nous percevons les TSS comme des travailleurs sociaux qui investissent un nouveau champ professionnel.

Prenant appui sur cette recherche, cet article présente et discute les modalités de division du travail entre TSS, médiateurs et enseignants, de même que la collaboration avec les directions d'établissement. Il éclaire la manière dont les TSS et les directions se positionnent face à ces modalités et se les approprient. Un regard est également porté sur la qualité perçue de la collaboration entre les différents acteurs scolaires et les défis qui se posent pour l'avenir.

\section{Méthodologie}

Cette recherche, de nature exploratoire et qualitative, a été menée auprès de TSS et de directions de CO francophones du canton de Fribourg, avec l'autorisation du Service de l'enseignement obligatoire de langue française (SEnOF).

La collecte des données a été réalisée à l'automne 2016 par le biais d'entretiens individuels semi-directifs (Blanchet/Gotman 2006). Nous avons réalisé neuf entretiens individuels ${ }^{4}$, six auprès de TSS et trois auprès de directions œuvrant dans sept CO fribourgeois. Les sujets, quatre hommes et six femmes, ont participé sur une base volontaire.

Deux guides d'entretiens - un à l'intention des TSS, l'autre des directions - ont été élaborés sur la base du cadre théorique de la recherche et en lien avec ses objectifs. Les thèmes abordés étaient identiques, mais les questions ont été adaptées en fonction des groupes d'acteurs interviewés. Les thématiques suivantes ont été abordées: a) le contexte institutionnel; 
b) les responsabilités, activités et compétences des TSS et des autres personnes ressources de l'école, de même que la spécificité de leur rôle; c) le fonctionnement des TSS et l'attribution des dossiers; d) la perception de la collaboration entre les différents professionnels.

Les entretiens ont duré en moyenne 1 h15 et ont tous été enregistrés avec l'accord des participants. Ils ont ensuite été retranscrits intégralement, puis soumis à une analyse thématique de contenu, réalisée dans un premier temps pour chaque participant, puis de façon croisée, sur la base d'un codage mixte effectué de manière semi-inductive (Bardin 2007).

\section{Résultats}

Les fonctions et missions des TSS

L'inscription du TSS dans la nouvelle loi scolaire et dans son règlement d'application a répondu à la volonté de la DICS et des établissements scolaires fribourgeois de promouvoir une certaine uniformisation des pratiques de soutien aux élèves en difficulté et d'«offrir une certaine égalité de traitement aux élèves et les mêmes prestations dans tous les CO du canton» (Dir1). Les directions ont été impliquées dans ce processus comme d'autres acteurs du canton (communes, inspecteurs scolaires, etc.) et il leur a été possible de «passer les articles en revue, on les a commentés, on a donné notre avis, on a demandé certaines modifications. On a été consultés.» (Dir3).

L'intégration du TSS a conduit à une réorganisation administrative du personnel de soutien, mais également à une reconnaissance officielle de sa fonction. Le TSS est désormais considéré comme un acteur faisant pleinement partie de l'encadrement scolaire, même si « le projet d'avoir un $100 \%\left[\mathrm{EPT}^{5}\right]$ de travail social pour 1000 élèves» (TSS1, TSS2) initialement prévu dans la loi est appliqué de manière très hétérogène sur le terrain. Dans les CO consultés, le taux d'encadrement oscille entre $39 \%$ et $124 \%$ EPT pour 1000 élèves, en fonction des besoins des écoles, du nombre et des problématiques des élèves à prendre en charge, de leur culture de la gestion des mesures de soutien internes à leur propre établissement entre médiateurs et TSS ainsi que des ressources financières engagées par les communes, en complémentarité avec l'Etat, pour rémunérer le poste du TSS. Selon un directeur,

Il y a une réelle volonté de généraliser l'intervention des travailleurs sociaux dans les CO. [...] Mais après, ce qu'il faut aussi savoir, c'est que tous les cycles d'orientation n'ont pas les mêmes besoins, n'ont pas la même population d'élèves et n'ont pas le même nombre d'élèves. (Dir3) 
Ainsi, les pratiques d'engagement des TSS dans les écoles varient en fonction des besoins, des subventionnements actuels et de l'incertitude liée aux financements cantonaux et communaux à venir (Von Aarburg 2014), ce qui soulève la question de la précarité des postes de TSS.

Le descriptif de fonction a été redéfini, en l'ajustant à celui qui existait déjà pour la partie germanophone du canton. Ce travail s'est fait de manière participative avec un groupe de «coordination TSS» qui s'est créé en 2010 «composé de responsables d'établissements, directeurs de CO et TSS [...], [dont] le sens a été de coordonner nos actions, de penser le travail social en milieu scolaire, de pouvoir expliquer ce qu'on fait, aussi bien à l'interne qu'à l'extérieur de l'école, de faire un travail pour clarifier les mandats.» (TSS3). La participation des TSS à ce groupe leur a permis de partager leurs réalités du terrain et de faire reconnaitre leurs fonctions, responsabilités et activités au quotidien. Ces échanges ont abouti à la constitution d'un descriptif de fonction commun qui définit le rôle et le mandat du TSS, à savoir "favoriser le contexte d'apprentissage et l'intégration sociale de l'élève.» (TSS3). Le rôle principal du TSS est donc de conseiller et soutenir les élèves en difficulté dans leur parcours ainsi que d'entreprendre des démarches de prévention et d'intervention précoce pour «éviter que cela devienne un gros problème» (Dir2). De par sa fonction, le TSS est amené à «créer des liens entre les acteurs de l'école» (Dir3), à soutenir et conseiller les enseignants et la direction dans le suivi des élèves en difficulté et à collaborer avec les autres professionnels du soutien (médiateur, psychologue, logopédiste). Le travail du TSS est orienté aussi vers l'extérieur du CO, en soutenant les familles des élèves en difficulté ainsi qu'en collaborant avec les acteurs du réseau professionnel (police, service de l'enfance et de la jeunesse, etc.). Un directeur insiste sur le fait que le TSS assume "un rôle social et éducatif» (Dirl), mais n'exerce pas de tâches pédagogiques.

Les entretiens mettent en évidence le développement d'une modalité de division du travail basée sur la différenciation dans tous les établissements scolaires consultés. Cela implique une distinction, sur le plan formel, entre les TSS, les médiateurs scolaires et les enseignants: chaque profil professionnel exerce sa fonction et ses missions et possède son cahier des charges, avec des responsabilités et activités prescrites distinctes. Malgré un cahier des charges clair, plusieurs directions estiment que l'arrivée du TSS a généré des incertitudes auprès des enseignants et médiateurs scolaires, concernant leurs rôles, responsabilités et activités. D’une part, les enseignants, tout en pouvant passer plus de temps à enseigner qu'à éduquer et s'occuper des élèves en difficulté, ont craint des ingérences des TSS 
dans leur travail avec les élèves. D’autre part, la similitude du travail avec les TSS a soulevé chez les médiateurs scolaires des questionnements relatifs à leurs tâches futures et à la division du travail avec les TSS. Une direction souligne que cela a nécessité des clarifications sur les plans institutionnel et informel entre TSS, enseignants, médiateurs:

Au départ, si on n'a pas de travailleurs sociaux, mais on a des médiateurs, ilya besoin d'une visibilité, d'une explication. Qu'est-ce qu'elle fait cette personne? Est-ce qu'elle va venir dans mon cours regarder mes élèves, me dire comment je dois faire dans mon enseignement, etc. ? Il y a plein d'éléments quand même qui déstabilisent [...]. (Dir3)

\section{L'intervention des TSS dans le CO}

Lorsqu'on interroge les TSS sur la nature de leur travail, la proximité avec le cahier des charges est frappante: il y a peu de décalage entre prescription et réalité. Ainsi, l'idée de placer l'élève au centre de leurs interventions apparaît de manière prioritaire. Le fait de devoir les articuler à la fois à l'interne de l'école avec les enseignants et les directions et avec les professionnels extérieurs à l'école est également une réalité du métier.

\section{Un travail centré sur l'élève et sa famille}

Le TSS centre son activité professionnelle sur l'élève en difficulté afin de "les soutenir, favoriser leur intégration sociale, accompagner un élève, faciliter le travail d'apprentissage de l'ensemble de la classe» (TSS3). Il est sollicité dans des situations complexes, "les situations qui ont besoin d'être traitées en réseau, puis d'être travaillées avec les familles» (TSS5), en particulier lorsque les enseignants sont face à un élève dont les difficultés ne relèvent pas que du comportement ou de la réussite scolaire: "s'ils ne savent plus quoi faire avec un élève qui sort des limites, ils viennent chez moi pour me demander" (TSS4). Les principales questions abordées par les TSS dans le suivi des élèves concernent la sphère scolaire ("problèmes de comportement en classe», "conflits avec les enseignants», etc.), leur situation personnelle («difficultés d'apprentissage», violence, "consommation de stupéfiants», etc.), la cellule familiale («maladie», «conflits» avec les parents, etc.) et les difficultés sociales ("problèmes d’intégration», précarité, "harcèlement scolaire», etc.). Cette réalité du travail du TSS, « un petit peu pompier, un petit peu infirmier» (TSS6), situé en première ligne pour intervenir auprès d'élèves spécifiques coïncide avec le regard des directions qui pensent que le TSS est vraiment « une aide lorsqu'il y a une problématique avec un élève en 
particulier [...]» (Dir1). La complexité des situations difficiles de certains élèves implique pour le TSS une collaboration étroite avec leurs parents: « $i l$ faut vraiment voir les parents de manière régulière, voir ce qu'il se passe et puis mettre en place un dispositif plus complexe» (TSS5). La relation entre l'école et la famille apparaît ainsi comme centrale dans l'intervention du TSS.

La différence entre le travail des médiateurs scolaires et des TSS concerne principalement la complexité de la situation des élèves, ce qui engendre des spécificités de chacun en termes de rôles, responsabilités et activités. Ainsi, le travail de ces acteurs «sont complémentaires et répondent à un réel besoin dans l'école» (TSS3). Les médiateurs interviennent davantage auprès d' «élèves qui rencontrent des difficultés passagères" (Dir2), au niveau de la classe, lorsqu'il y a des conflits entre élèves, une détérioration du climat ou encore des problèmes relationnels avec les enseignants. Les TSS s'occupent davantage des «cas qui n'ont pas pu être gérés avant» (TSS1, TSS2), notamment lorsqu'il y a la nécessité de travailler avec les réseaux familial et professionnel qui entourent l'élève en difficulté. En ce sens, la diversité et la complémentarité des regards, des expertises et des compétences permettent d'offrir un soutien différencié aux élèves, enseignants et parents.

Une proximité en construction avec les enseignants

L'accompagnement des élèves en difficulté implique une collaboration étroite entre TSS et enseignants afin de partager leurs expériences et compétences et construire ainsi une intervention qui réponde aux besoins des élèves: "Ce travail consiste à soigner le lien, à savoir communiquer sur une situation d'élève [...] tout en étant respectueux» (TSS3). Cette collaboration présente des avantages aussi pour le travail des enseignants: Il faut

faire tout le travail de coulisses qui va être utile pour que l'enseignant, quand il donne son cours, puisse le faire dans les meilleures conditions possibles. (TSS5)

Les TSS insistent sur la nécessité de cette proximité avec les enseignants qui se joue dans les rencontres formelles, mais surtout à travers des échanges informels:

Je vais à la cafétéria et à la salle des maîtres [...]. Je suis un pèlerin dans cette école, je bouge, je travaille beaucoup dans les couloirs et dans les lieux où sont les gens. (TSS6) 
Pour les TSS, ce lien se construit petit à petit et nécessite de se montrer, de dire qu'on existe: "un des aspects de mon travail [...] c'est la «publication de moi-même»» (TSS4). Il est en outre indispensable de gagner le respect et la confiance des enseignants.

Selon les TSS, la plupart des enseignants perçoivent les atouts de cette collaboration pour le bien-être des élèves en difficulté et pour le soutien que ces derniers peuvent leur apporter:

Ils se rendent compte de l'avantage de venir avant, de se dire "Elles sont là.» Et il y a aussi une question de temps à disposition. On est là à $100 \%$ "Ah, elles sont là, elles peuvent prendre le temps. Elles ont les compétences pour pouvoir gérer des situations complexes. » (TSS1, TSS2)

D’autres enseignants ne travaillent pas spontanément avec eux: soit ils sollicitent leur soutien uniquement pour se débarrasser des cas difficiles, soit ils pensent devoir résoudre eux-mêmes les problèmes. Les TSS font l'hypothèse que certains enseignants ne font pas appel à leurs services en raison de divergences dans les cultures et pratiques professionnelles ou par peur d'être remis en question: "ils se sentent, ils nous le disent, attaqués, jugés, alors qu'on n'est pas dans ce regard-là» (TSS1, TSS2). Un directeur confirme que cette collaboration est parfois compliquée

[...] en fonction de la sensibilité de certains enseignants dès qu'il y a une intervention de la travailleuse sociale, cela va peut-être mettre en lumière certains comportements et réactions de l'enseignant. (Dir2)

Une autre difficulté concerne le temps: le TSS permet de soulager le travail des enseignants dans la gestion des problèmes sociaux qui prennent beaucoup de temps et sortent de leur champ de compétences, alors que les enseignants hésitent parfois à s’impliquer, car l'intervention des TSS est considérée comme chronophage:

Un élève qui est suivi, elle [la TSS] propose des rencontres, donc c'est des temps de réunion, des séances de réseau, de voir les objectifs et puis ça prend du temps. Donc il y a aussi une limite au niveau de la charge de travail de l'enseignant. (Dir2)

En ce sens, la relation entre TSS et enseignants apparaît comme un champ de tension où se mêlent nécessité de trouver des solutions, intérêt pour 
lâcher ou prendre en main des situations, reconnaissance réciproque, collaborations indispensables et le maintien des rapports de pouvoir entre les groupes professionnels.

Selon les TSS les spécificités de leur fonction et intervention sont parfois peu comprises et (re)connues par les enseignants. Par ailleurs, la réticence de certains enseignants à collaborer témoigne des enjeux au niveau de la définition, voire du contrôle des espaces spécifiques d'intervention des deux acteurs au sein de l'école ainsi que de la présence de relations de concurrence entre ces profils professionnels. Ces difficultés sont probablement liées à la diversité des cultures et formations des enseignants et des travailleurs sociaux qui entrainent une vision différente de l'élève, de leurs mandats respectifs (instruire versus éduquer) ainsi que de leur intervention. La situation est susceptible d'évoluer car la recherche a été menée en 2016-2018 alors que plusieurs TSS étaient récemment arrivés dans les $\mathrm{CO}$.

Finalement, les entretiens montrent que l'introduction des TSS dans les CO soulève deux enjeux majeurs concernant la cohabitation avec les enseignants. Le premier est lié à la reconnaissance de la place de chaque figure professionnelle dans l'école ainsi qu'à la connaissance et la compréhension mutuelle des responsabilités, activités et compétences, qui vont de pair avec la nécessité pour les TSS de gagner de "la crédibilité des enseignants, de la direction de l'établissement et de toute la hiérarchie jusqu'aux politiques» (TSS1, TSS2). Le deuxième enjeu concerne d'une part les ressources mises à disposition des professionnels (espace, temps) pour leur permettre de s'approprier des cultures et pratiques professionnelles respectives, que ce soit en termes de "langage», d'intervention, de mode de travailler, de façon de collaborer ainsi que de rythme de l'action, d'autre part les mécanismes de coordination et communication. A ce propos, deux TSS précisent que

dans le social, on est dans une culture de collaboration pluridisciplinaire, d'apprendre à se transmettre les situations, de pouvoir en échanger. Dans la culture scolaire, ils sont individualistes, surtout dans un CO. [...] ils sont seuls maîtres à bord sur leur bateau. (TSS1, TSS2)

Quoi qu'il en soit, tous les TSS soulignent une évolution positive depuis leur engagement dans l'école que ce soit sur le plan de la reconnaissance de leurs spécificités par les enseignants que sur celui de leurs relations caractérisées de plus en plus par la collaboration: 
Il y a du terrain qui se gagne dans les collaborations avec les enseignants. [...] Il y a des messages qui ont été passés il y a quatre ans qui commencent à être incarnés aujourd'hui. (TSS1, TSS2)

Des liens étroits avec les directions des CO

Les TSS collaborent également avec la direction, en particulier lorsqu'il y a des décisions à prendre par rapport à certains élèves: "Avec l'équipe de direction, [les TSS] ont aussi un rôle de décision, un rôle de signalement, ils ont des responsabilités propres à leur fonction [...]» (TSS3). Ces contacts réguliers permettent d'échanger sur l'observation, l'analyse et l'évolution de la situation des élèves difficiles ainsi que de mettre sur pieds des interventions appropriées à leurs besoins :

Les adjoints de direction arrivent avec leurs situations, les présentent pour que nous soyons au courant et la travailleuse sociale l'entende aussi. Et puis comme ça [...] on peut tout de suite savoir si c'est une situation pour elle ou pas. (Dir1)

Cette étroite collaboration permet une grande fluidité en termes de fonctionnement et suivi des situations:

Toutes les semaines, elle entend ces situations et puis c'est aussi l'occasion pour nous d'avoir des retours, [qu'] elle nous dise ce qui a été fait par rapport à ces situations. (Dir1)

Tout en étant perçue positivement, la collaboration entre TSS et direction " a amené parfois à devoir confronter des points de vue» (TSS3), les directions prenant davantage en considération "l'aspect fonctionnement de l'école, etc.» (Dir2) alors que les TSS étant plus focalisés sur "l'aspect personnel, humain» dans la prise en charge de l'élève (Dir2).

Une mobilisation des réseaux autour des élèves

En dehors du TSS avec les acteurs de l'école, nos répondants insistent sur l'importance des relations avec la famille et le réseau professionnel qui entourent l'élève en difficulté ainsi qu'avec les instances officielles qui touchent de près ou de loin à la jeunesse. En élargissant la problématique de l'élève pour "donner le point de vue plus social de ce qui se passe à l'école» (Dir3), le TSS est ainsi amené à aller vers l'élève en difficulté et son entourage (scolaire, familial et social) pour les mettre autour de la table et trouver 
des solutions. Nos participants soulignent la polyvalence des TSS qui, sans être juristes, psychologues, enseignants, doivent connaître beaucoup d'aspects de ces différents métiers pour réussir à les faire dialoguer et résoudre des situations complexes:

La profession de travailleuse sociale est très diversifiée. C'est une personne qui n'est pas une spécialiste, mais qui est polyvalente et qui doit posséder un certain nombre de qualités. (Dir3)

Par ailleurs, la connaissance des réseaux professionnels tissés au fil des ans constitue une des spécificités du travail du TSS aux yeux des directions, en apparaissant comme un allié qui dispose de ressources que les autres n'ont pas.

\section{Discussion}

Un métier avec de multiples facettes et une délimitation claire du travail

La présence des TSS implique, pour les directions des CO, de reconsidérer les missions et rôles des autres acteurs du soutien et de repenser l'organisation du travail entre TSS, enseignants et médiateurs.

Nos données montrent une grande variété des responsabilités et activités du TSS en fonction des écoles et de son taux d'engagement. Les activités du TSS se caractérisent à la fois par un travail dans l'urgence axé sur des situations complexes d'élèves et par des actions de prévention précoce et développement du vivre ensemble, en particulier dans les CO qui bénéficient de dotations plus importantes en TSS. Ces premiers résultats font écho aux constats déjà identifiés dans les différents cantons par Iseli / Grossenbacher-Wymann (2013), et Abry Kalenga et ses collègues (2015). D'une manière générale, nos répondants sont favorables à une augmentation de la présence des TSS au sein des écoles afin de leur permettre de mieux répondre aux besoins spécifiques des élèves (Wattendorff / Landry 2012), de participer au développement d'un meilleur climat pour l'ensemble des élèves et enseignants ainsi que de gagner en visibilité.

Les résultats mettent également en évidence les spécificités des fonctions de TSS, d'enseignant et de médiateur scolaire, de même que les zones de tuilage entre elles, en termes de rôles, responsabilités, activités, compétences. Ainsi, la division du travail entre les TSS et les autres acteurs de l'école semble clair (voir Figure 1). Les responsabilités et activités des uns et des autres s'articulent autour des finalités de l'école (exprimées dans la nouvelle loi scolaire fribourgeoise et son règlement ainsi que dans le plan 
d'études) liées à l'éducation (développement du rapport à soi, aux autres et au monde) et à l'instruction (acquisition des connaissances et des compétences, régulation et évaluation) des élèves, en parallèle au mandat implicite de sélection opéré par le système scolaire. Ces responsabilités et activités s'expriment dans des situations collectives ou individuelles.

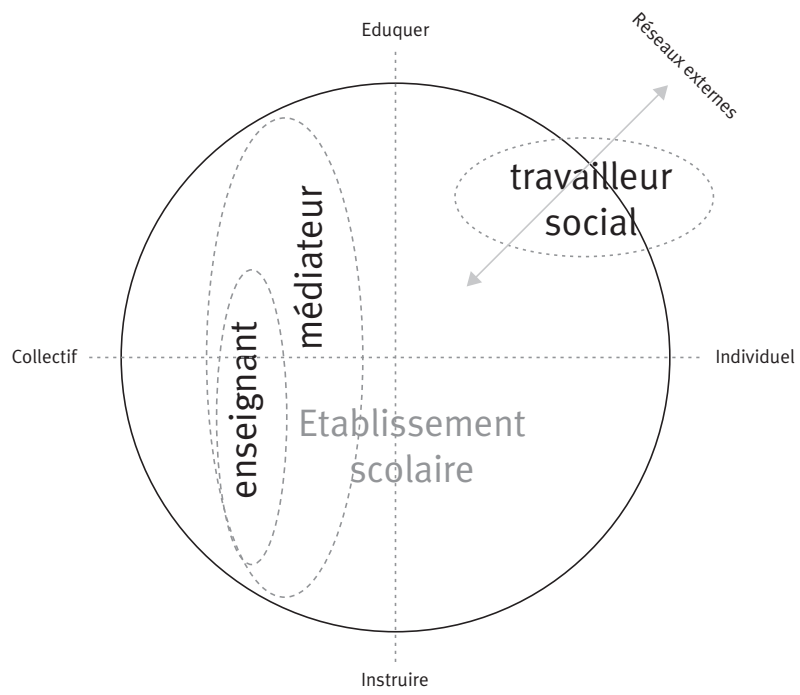

D’une manière générale, les enseignants servent en premier lieu l'objectif d'instruction et de qualification des élèves, alors que les TSS s'attachent prioritairement aux buts éducatifs et de socialisation attribués à l'école. Pour réaliser ces missions, les enseignants gèrent le collectif d'élèves dans les classes et travaillent davantage dans une logique solitaire, alors que les TSS prennent en charge de manière plus individualisée les jeunes en difficulté qui leur sont confiés, tout en collaborant avec les autres acteurs de l'école ainsi qu'avec les familles et le réseau de professionnels qui les accompagnent. La logique de «la classe et les élèves pour les enseignants» et du «jeune en difficulté pour le TSS» fonctionne bien et structure le travail de chacun. Les médiateurs scolaires, présents dans les CO consultés, se trouvent dans une position intermédiaire en abordant des problématiques éducatives dans le contexte collectif de la classe.

Cette articulation entre éducation et instruction est bien entendu plus subtile qu'il n'y paraît et même si les aires de travail de chacun semblent 
bien délimitées, ces deux enjeux se croisent inévitablement. D’une part, la dimension éducative est également l'affaire des enseignants lorsque l'élève dysfonctionne et, par son comportement, remet en question l'institution scolaire ou la classe et son fonctionnement. Avant d'instruire, l'école - en tant qu'institution - doit être instituée (Meirieu 2004). D’autre part, les apprentissages, la réussite scolaire et les conséquences que cette dernière entraîne en termes d'orientation et sélection préoccupent également les TSS. Les raisons d'un dysfonctionnement chez un élève sont multifactorielles et la réussite scolaire doit être prise en compte par les TSS, à plus forte raison si elle s'inscrit dans des contextes où les problématiques d'inégalités sociales sont présentes. Ces éléments renvoient aux ajustements des enseignants et des TSS par rapport à la situation d'un élève.

Finalement, l'introduction des TSS dans les CO est perçue tant par les TSS que les directions comme un plus pour le bien-être des élèves, le climat de l'école et le travail des enseignants (Wattendorff / Landry 2012). La diversité et la complémentarité des regards, des métiers, des compétences entre enseignants, TSS et médiateurs permettent en effet une prise en charge différenciée et complexe des besoins des élèves.

\section{Une dynamique relationnelle qui se joue entre intérieur et extérieur de l'école}

Cette bonne compréhension des rôles de chacun ne préserve pas des tensions. En effet, les TSS doivent faire leur place dans ce nouveau champ professionnel et susciter des dynamiques relationnelles avec différents partenaires, que ce soit à l'intérieur ou à l'extérieur de l'école.

Dans les CO fribourgeois, les TSS entretiennent des relations étroites avec les directions, notamment en raison des situations particulières des élèves. En ce sens, la coopération entre école et travail social se passe selon le modèle intégré décrit par Hafen (2005) et Kottelat (2015). Cette proximité du TSS avec l'autorité de contrôle et son indépendance du corps enseignant peuvent occasionner une certaine méfiance, voire des craintes de la part des enseignants, ces derniers pouvant hésiter à se confier aux TSS et renoncer à leur faire part de leurs observations ou sentiments pour éviter tout jugement. La confiance entre TSS et enseignants ne se décrète pas et doit donc se construire dans un réel partenariat à l'interne de l'école : ces contacts sont habituellement informels et concernent souvent des sujets personnels et délicats (bien-être d'un élève, attitudes de l'enseignant, etc.). Ce patient tissage de liens nécessite du temps et de la disponibilité pas toujours compatibles avec les conditions de travail, les taux d'activités insuffisants des TSS et la disponibilité des enseignants en dehors de la classe 
(Wattendorff / Landry 2012). Cette collaboration est toutefois jugée comme déterminante par les TSS, car même si ce sont eux qui prennent en charge les situations des élèves en difficulté, ce sont les enseignants qui, la plupart du temps, sont en première ligne pour identifier les premiers troubles des élèves. Ce passage de témoin entre enseignants et TSS repose sur la reconnaissance mutuelle des fonctions et missions exercées au sein de l'école et sur la compréhension réciproque d'une ligne de démarcation définissant les espaces spécifiques d'intervention de chacun. Cette compréhension se base sur la culture du dialogue qui évite que les uns et les autres se sentent dépossédés de leur mission ou, au contraire, se croient investis de problèmes qu'ils estiment ne pas devoir prendre en charge.

D'autre part, les TSS sont également un relais important entre l'intérieur et l'extérieur de l'école. Ce statut s'actualise en particulier lorsqu'il s'agit de mobiliser la famille de l'élève en difficulté ainsi que le réseau de services spécifiques qui l'accompagnent. Le TSS apparaît ainsi comme une figure de l'école, capable de mobiliser des ressources externes pour aider l'élève en articulant les besoins du jeune avec les impératifs scolaires.

\section{Conclusion}

Cette recherche fournit une photographie de la manière dont s'opère la collaboration entre TSS, enseignants, directions et médiateurs scolaires dans les CO francophones du canton de Fribourg, ceci à partir des regards croisés des TSS et des directions.

Les résultats ont mis en évidence la polyvalence du métier de TSS et les compétences que cela implique. Ainsi, la légitimité du TSS s'acquiert, au fil des ans et des expériences, à travers son aptitude à jouer le rôle de trait d'union entre les acteurs de l'école et les partenaires externes, arrivant ainsi à «se faire une place» dans un domaine qui, à l'origine, n'était pas un de ses champs d'intervention habituels. Par ailleurs, le développement d'une modalité de division du travail basée sur la différenciation entre TSS, médiateurs scolaires et enseignants a été observée dans tous les établissements scolaires. Toutefois, même si les rôles de ces trois acteurs apparaissent comme bien distincts, la prise en charge des élèves en difficulté les mobilise tous: il s'agit notamment de prendre en compte les situations personnelles complexes de ces jeunes, tout en gérant la progression de leurs apprentissages et en préservant une certaine équité avec les autres élèves. Ces situations nécessitent que tous les acteurs de l'école dépassent la simple information sur des procédures de signalement ou des modalités de prise en charge ou encore l'application stricte des cahiers 
des charges. Il est important de créer une dynamique d'établissement où l'élève est placé au centre des préoccupations de tous les acteurs et où chacun, à son échelle et dans des proportions variables, se sente responsable de cette double mission: éduquer et instruire. Toutefois, les représentations réciproques des missions et savoir-faire pouvant jouer un rôle dans la collaboration entre enseignants, médiateurs et TSS, il serait souhaitable que les directions des établissements scolaires réalisent, dès l'engagement des TSS, un travail formel d'explicitation de la structure organisationnelle de l'école (organigramme, rôles, cahiers des charges, répartition des responsabilités et activités) et qu'elles mettent à disposition des professionnels des ressources (organisation du temps et de l'espace du travail) pour des échanges et de la transmission d'informations afin que chacun trouve sa place dans l'école. De même, une formation continue commune pourrait être envisagée pour les enseignants et les TSS, afin de les sensibiliser aux cultures et pratiques professionnelles respectives et d'éviter ainsi les écueils d'une communication défaillante ou un éventuel désengagement de ces acteurs. Il s'agirait également d'une bonne opportunité pour permettre à ces professionnels de faire face aux nouveaux défis que l'école d'aujourd'hui doit relever. Ils pourraient ainsi profiter de la pluralité et de la vivacité des contextes dans lesquels ils agissent pour continuer à se construire et se développer (Conq 2010).

Les résultats de cette recherche doivent être interprétés avec précaution, d'abord en raison de la taille limitée de l'échantillon, de sa non-représentativité et de sa constitution sur la base du volontariat. Ensuite, les éléments relevés concernant la division du travail, le positionnement des acteurs et la collaboration reposent uniquement sur le discours d'une partie des acteurs. Finalement, la recherche a analysé la collaboration entre les TSS et les autres acteurs de l'école seulement quelques années après l'introduction du TSS dans les écoles; il serait aujourd'hui intéressant d'en suivre l'évolution.

\section{Bibliographie}

Abry Kalenga, Jacqueline; Cusin Girod, Christine; Gendre, Florence \& Sciboz, Luc (2015). Travail social en milieu scolaire Fribourg Ville. Rapport d'activité 2014-2015.

Aebischer, Camille (2017). Quels enjeux les enseignant.e.s des cycles d'orientation fribourgeois identifient-ils dans le cadre de la collaboration avec les travailleurs et travailleuses sociales en milieu scolaire? Travail de Bachelor Haute école de travail social Fribourg, Givisiez.

AvenirSocial (2016). Lignes directrices $d u$ travail social en milieu scolaire. Berne: AvenirSocial. 
Bardin, Laurence (2007). L'analyse de contenu. Paris: Presses Universitaires de France.

Bercot, Régine; Divay, Sophie \& Gadéa, Charles (Dir.) (2012). Les groupes professionnels en tension. Toulouse: Octares.

Blanchet, Alain \& Gotman, Anne (2006). L'entretien. Paris: Armand Colin.

Bohrer, Isabelle (2009). Le travail social en milieu scolaire. Un vrai boom ... et un défi pour Avenir-Social. In: Actualité Sociale, 21, p. 19-20.

Buhler, Clothilde (2015). Un binôme devenu nécessaire. In: ActualitéSociale, 55, p. 3.

Cattin, Cindy \& Sottas, Stéphanie (2014). Le travail social en milieu scolaire: une intervention face à l'absentéisme scolaire. Travail de Bachelor Haute école de travail social Fribourg, Givisiez.

Chaignat, Ophélie \& Jacopin, Lorraine (2015). Conseillers école-famille: regards sur leur collaboration avec les parents. Travail de Bachelor Haute école de travail social Fribourg, Givisiez.

Changkakoti, Nilima \& Akkari, Abdeljalil (2008). Familles et écoles dans un monde de diversité: au-delà des malentendus. In: Revue des sciences de l'éducation, 34(2), p. 419-441.

Chevalier, Sarah (2015). La prévention et la promotion de la santé dans le milieu scolaire: les actions professionnelles des travailleuses et travailleurs sociaux en milieu scolaire dans le canton de Neuchâtel. Travail de Bachelor Haute école de travail social Fribourg, Givisiez.

Conq, Nathalie (2010), Introduction. In: Nathalie Conq, Jean-Pierre Kervella,
Alain Vilbrod (sous la dir. de), Le métier d'éducateur spécialisé à la croisée des chemins. Paris: L'Harmattan, p. 11-19.

Demazière, Didier \& Gadéa, Charles (Dir.) (2009). Sociologie des groupes professionnels. Acquis récents et nouveaux défis. Paris: La Découverte.

Dubar, Claude; Tripier, Pierre \& Boussard, Valérie (2011). Sociologie des professions ( $3^{\text {ème }}$ éd.). Paris: Armand Colin.

Hafen, Martin (2005). Soziale Arbeit in der Schule zwischen Wunsch und Wirklichkeit. Ein theoriegeleiteter Blick aufein professionelles Praxisfeld. Luzern: interact.

Iseli, Daniel \& Grossenbacher-Wymann, Simone (2013). Travail social en milieu scolaire. Lignes directrices (3 ${ }^{\text {ème }}$ éd.). Berne: Direction de l'instruction publique.

Kottelat, Jacques (2015). Les travailleurs sociaux sont de plus en plus nombreux en milieu scolaire. In: ActualitéSociale, 55, p. 7-8.

Meirieu, Philippe (2004). Faire l'école pour faire la classe. Paris: ESF.

Vezinat, Nadège (2016). Sociologie des groupes professionnels. Paris: Armand Colin.

Von Aarburg, Hans-Peter (2014). «Médiation scolaire» et «travail social en milieu scolaire». Le cas d'un positionnement professionnel. In: Cultures et Sociétés, 30, p. 48-54.

Wattendorff, Matthias \& Landry, Christine (2012). Enquête sur le travail social en milieu scolaire en ville de Fribourg (SSA FR). Rapport final.

\section{Annotations}

1 Les descriptifs de fonctions du médiateur scolaire et du TSS peuvent être consultés sur le site de l'Etat de Fribourg : https:// www.fr.ch/dics/formation-et-ecoles/415-ans/mediation-et-travail-social-enmilieu-scolaire.
2 Il existe différents modèles de collaboration entre travail social et école au niveau de l'organisation et de la coopération entre les TSS et les autres acteurs : le modèle intégré (ou d'intégration) qui implique une inclusion du TSS au niveau de l'organisation et/ou une coordina- 
tion étroite du TSS avec la direction et les enseignants et le modèle additionné (ou de subordination) selon lequel le TSS ne fait pas partie de l'organisation interne de l'école et/ou les objectifs et activités ne sont pas déterminés et coordonnés de manière commune avec les autres acteurs. Des variantes sont possibles entre ces deux pôles (Hafen, 2005; Kottelat, 2015).
3 A titre d'exemple, en 26 numéros, la revue des Hautes écoles pédagogiques de Suisse romande Formation et pratiques d'enseignement en questions ne présente aucun article scientifique traitant de cette question ces quinze dernières années.

4 A noter qu'un entretien avec les TSS a été mené en binôme.

5 Equivalent plein temps (EPT).

\section{Notes biographiques}

Alida Gulfi, docteure en Science de l'éducation à l'Université de Fribourg, est professeure à la HES-SO Haute école de travail social Fribourg. Ses recherches récentes s'intéressent à la collaboration entre familles migrantes d'enfants en situation de handicap et professionnels, l'articulation entre professionnels de l'éducation sociale, la collaboration interprofessionnelle social-santé, le rapport à l'autre chez les étudiants en travail social et ergothérapie ainsi que l'insertion professionnelle. Lien vers la liste des publications : https://people.hes-so. ch/fr/profile/alida.gulfi.
Pierre-François Coen, titulaire d'un doctorat en Science de l'éducation de l'Université de Fribourg, est engagé comme chercheur et formateur à la Haute école pédagogique de Fribourg et à la Haute école de musique Vaud - Valais - Fribourg. Ses travaux de recherche s'inscrivent dans les problématiques liées à la formation des enseignants, plus spécifiquement à l'analyse des processus d'autorégulation et d'autoévaluation en formation, d'intégration pédagogique des technologies numériques et d'éducation artistique. Lien vers la liste des publications : https://www.hepfr.ch/ users/coenpedufrch. 